\title{
Evaluating the Deployment of a Mobile Technology in a Hospital Ward
}

\author{
Charlotte Tang \& Sheelagh Carpendale \\ Department of Computer Science \\ University of Calgary \\ Calgary, Alberta, Canada T2N 1N4 \\ char.tang/sheelagh@ucalgary.ca
}

\begin{abstract}
Since health care teams are often distributed across time and location, information sharing is crucial for effective patient care. Studying the use of a mobile information technology in a local hospital ward at two months and eleven months after its deployment identifies both shortand long-term phenomena and reveals a mismatch between the intentions behind the deployed mobile technology and the nurses' current work practices. We contrast the new mobile technology with the paper artifacts that were previously relied upon in nursing work. Finally, in light of these findings, we suggest design directions for future technology to support the nursing shift work.
\end{abstract}

\section{Author Keywords}

Observation, shift change, information flow, mobile technology, computer-on-wheels, personal notes.

\section{ACM Classification Keywords}

H.m. Information Systems: Miscellaneous.

\section{INTRODUCTION}

Health care, as commonly practiced in hospitals, is a team effort distributed across time and location. One of the important factors for effective patient care is the sharing of information. That is, medical practitioners need to have access to the right information, at the right time, and ideally in the right location. We examine the sharing of information across nurses' shift change as one of the important factors in this process. The distributed nature of medical work entails the need for mobility to ensure that clinicians, who need to be in different places at different times, have access to information, use shared resources and are able to reach specific personnel $[1,13]$. One response among hospitals is to shift from paper documents to electronic health records (EHR) to pave the way for distributed information sharing.

Permission to make digital or hard copies of all or part of this work for personal or classroom use is granted without fee provided that copies are not made or distributed for profit or commercial advantage and that copies bear this notice and the full citation on the first page. To copy otherwise, or republish, to post on servers or to redistribute to lists, requires prior specific permission and/or a fee.

CSCW'08, November 8-12, 2008, San Diego, California, USA

Copyright 2008 ACM 978-1-60558-007-4/08/11...\$5.00.
Nevertheless, if information can only be accessed at stationary points such as immobile desktop computers, information sharing is tied to these designated locations. This defeats the potential benefits of real-time sharing of information at points of care, which may provide improved patient-centered services and resource utilization. This drives exploration of the potential of mobile information technology $[1,3,13,15]$. Theoretically, mobile technology has the potential to provide information access anytime and anywhere. Therefore, there is an increasing adoption of mobile information technology in the medical settings.

A technological setup was deployed in a local hospital ward to allow information access and data entry throughout the ward as a means to advance patient care. The deployed technology consists of a wireless mobile computer and a digital information system. We conducted an observational study to investigate the impact of this mobile technology on the information flow practices in nursing work. The goal of our study was to investigate how the mobile technology was used for information access and entry and if difficulties were encountered while using this technology. We were also interested in whether the mobile technology was successful in replacing the use of personal paper information artifacts which nurses had previously relied upon for carrying out their nursing tasks.

\section{RELATED WORK}

A hospital is an information-rich environment in which work is typically accomplished by cooperation among clinicians possessing different expertise. While carrying out the work required of a specific position, clinicians constantly assemble new information from an array of information sources, such as by assessing a patient's illness trajectory or by evaluating his/her lab reports, to dynamically shape treatment and/or care plans to best suit the patient's current condition. Meanwhile, they also need to disassemble the collected knowledge to appropriate information repositories such as a patient chart, ideally instantaneously. In this way, other clinicians may use the information to decide or adjust the treatment and care schedule accordingly. These information assembly and disassembly activities are important; communication failure among clinicians has been found to be a frequent 
contributing factor in many preventable adverse events to patients $[6,21]$. Therefore, these activities form an integral part of clinicians' daily work and require a high level of coordination and collaboration among them as well as timely availability of specific information artifacts $[6,17]$.

Traditionally in medical settings, information sources exist in a variety of media such as verbal, paper, and display medium [2,4,10,14,27]. Verbal communication is wellknown to be effective in interpersonal communication, particularly during face-to-face conversations with the use of visual aids like gestures $[3,11,25]$. Large displays such as whiteboards and bulletin boards are useful for broadcasting information, especially asynchronously, to a large audience. These displays are also effective in joint discussion and negotiation [26,27] and keeping an awareness and coordination of ongoing activities [2].

With increasing specialization in medicine, a patient may require synchronized care from multiple specialties and clinical professionals [6,9]. Hence paper-based information documents often have to be physically moved between locations where and when they are needed. While paper documents afford valuable benefits in viewing, reviewing, customizing, annotating, carrying, and amending data $[8,16,18]$, they often fall short in providing timely, locationindependent and simultaneous multiple information access. Therefore, in reality, required information may not be available at the right place and/or at the right time since tedious, sometimes complex, administrative policies, procedures, and coordination often impedes the physical delivery of paper information documents [8]. Thus, medical care is shifting towards the use of technology. Medical records are now largely digital, although most hospitals still maintain a certain amount of paper documents. In general, digital solutions have been increasingly replacing paper documents to provide more consistent, integrated, distributed, and timely sharing of information, which in turn improves work performance [7,18,19].

Mobile information tools, such as personal digital assistants (PDAs), wireless tablet PCs, and wireless mobile computers-on-wheels, have been widely adopted in the medical settings to allow ubiquitous access, and remote access to up-to-date patient information $[6,19]$. It was also found that clinicians were able to spend more time with their patients due to time savings when they did not have to physically move to search for needed information $[5,6]$.

In the remainder of the paper, we first briefly outline the studies that we have conducted, both before and after the mobile technology was deployed. Next, we describe the post-deployment study in detail and report our findings. We discuss the impact of this mobile technology on the use of paper personal notes in nursing work. Finally, we conclude with a description of design suggestions for supporting workplace mobility in the hospital setting.

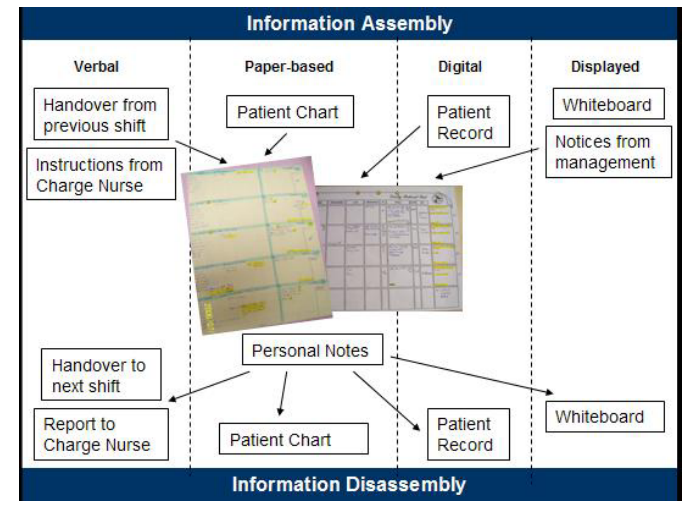

Figure 1. Transition of information through different media types in the information flow process

\section{STUDIES OF INFORMATION FLOW PRACTICES}

We conducted two field studies in a local hospital ward. Our first study, conducted six months before deployment of mobile technology, used minimally-intrusive observations of 42 nurses, interviews and examination of formal and informal information documents to thoroughly understand the basic information flow practices during the nurses' shift change. Information sharing took place as a pair of parallel processes: information assembly by incoming nurses and information disassembly by outgoing nurses, through four different media: paper, verbal, displayed, and digital media (Fig. 1) [21]. In this first study, digital information was only accessible through stationary desktop computers. We identified the flow of information between various information sources (e.g., paper-based patient chart and EHR) and paper-based personal notes prepared by nurses, customized at the beginning of their shifts by extracting important information from various information sources. Their shift work depended on these personal notes as their intermediate notepad and frontline information source.

Our second study was conducted in two stages, at two and eleven months after deployment of the mobile technology computer-on-wheels (COW) - to uncover short- and longterm phenomena. It is this second study (i.e. postdeployment study) that is discussed in this paper.

\section{POST-DEPLOYMENT STUDY}

In the long run, the goal of this mobile technology is to achieve a paperless hospital ward whereas the short term goal is to reduce the use of paper artifacts in the daily work flow of clinical work. As this technology was designed for nurses, the short term goal is to remove the paper-based artifacts primarily used by nurses before the technology adoption. These paper artifacts are the patient care summaries which were printed at the beginning of each nursing shift and the paper personal notes that nurses prepared at the beginning of their shift by gathering information from various distributed multimedia information sources (Fig. 1). 
We conducted the post-deployment study in two stages as difficulties are typically experienced in the early adoption of new technology. Thus, it is important to distinguish short- and long-term difficulties. The former will typically be resolved on its own, while the latter may require intervention for improvements. Therefore, the study was conducted at two months and eleven months after the technology was deployed. Although participants in the two stages varied slightly due to shift assignments, staff vacation, and turnover, the study design allows us to uncover short- and long-term phenomena. This revealed how the technology was adopted and how it could be improved.

\section{Setting}

The research site is an acute medical teaching unit in an urban hospital. Patients admitted to this ward are often transferred directly from ICU. Therefore, the patients generally still require acute care for a vast array of, often multi-system, illnesses. However, the nurse-to-patient ratio ranges from 1:4 to $1: 8$ depending on work shifts, as opposed to the drastically lower ratio of $1: 1$ or $1: 2$ in the ICU. Therefore, nurses working at this ward constantly face high stress and time pressure. Yet, these nurses are recognized for their enthusiasm towards their work and the strong dynamic team environment that they have built in such a high-stress and time-critical hospital ward. In addition, innovative research activities frequently take place on this ward. Therefore, nurses working on the ward are generally open-minded towards technology.

The ward is configured with a star design having a centrally located nursing station and four radiating ward wings of patient rooms. Its layout makes it convenient to access information at the central information hub but makes it hard for nurses working in different wings to communicate and to maintain awareness. Two wired desktop computers are available in each ward wing; nurses sit on high backless stools (Fig. 2). The computer terminal beside the nursing station is placed with seven wired desktop computers, equipped with comfortable padded chairs (Fig. 3).

The mobile device, a "computer-on-wheels" (COW), is a desktop computer placed on an ergonomically designed mobile cart running on a wireless network to provide

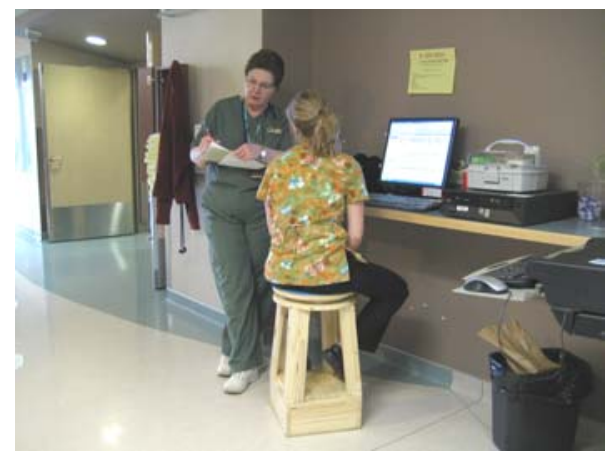

Figure 2. A Nurse working at a desktop computer in a ward wing

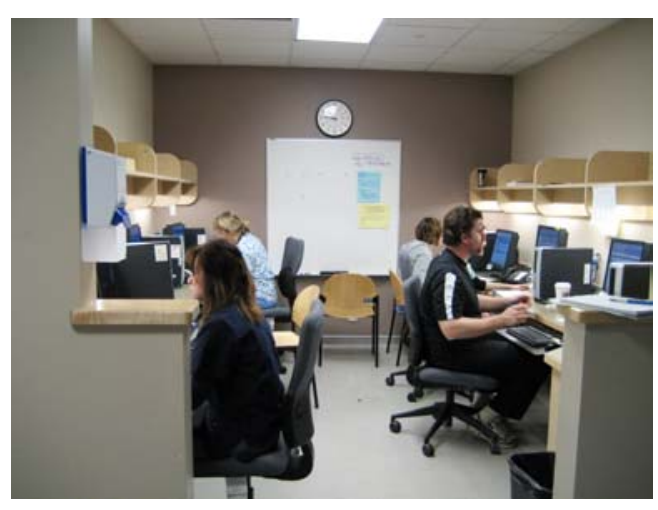

Figure 3. Nurses working at desktop computers in the computer terminal

mobile access to the EHR (Fig. 4). They allow clinicians to have immediate, and in many cases, bedside medical information, including high-resolution digital images, laboratory results, and medication histories, with a few mouse clicks. The input is primarily provided via keyboard entry and mouse manipulation. The height of the cart, i.e. the height of the keyboard, is slightly adjustable for standing or sitting on a high stool (Fig. 4).

\section{Method}

In both stages of the study, we used minimally intrusive observations, informal interviews and examination of the information documents to investigate how nurses use the mobile device for information flow and to explore if difficulties were encountered. Informal interviews were conducted when clarification and elaboration of their actions were needed and when the situation allowed. We completed the study with a questionnaire survey. The purpose of the survey was to gather qualitative and quantitative feedback from participants regarding the benefits and drawbacks they experienced from the technology after using it for almost a year. We provided the questionnaire in the staff room during participants' meal breaks. Respondents voluntarily participated in completing the questionnaire. No personal identification information was asked in the questionnaire and the completed questionnaires were randomly put in a large envelop to preserve their anonymity. We received 29 questionnaires.
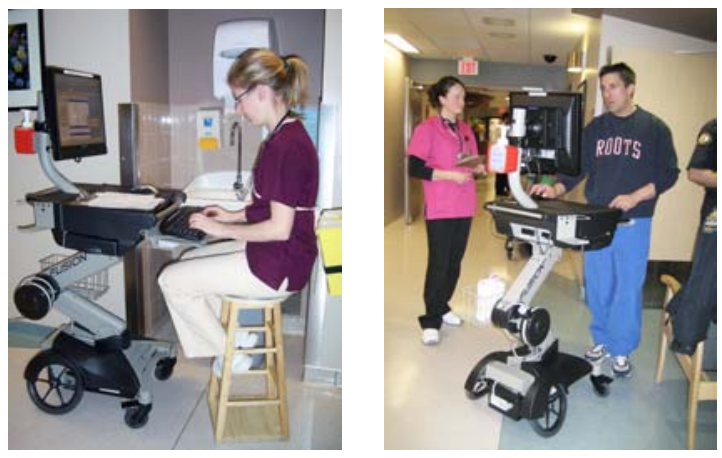

Figure 4. Nurses working at wireless COWs in their ward wings 
Each participant spent about 15 minutes on the questions which consisted of a variety of multiple choice, and some short and longer answer open-ended questions. Our multiple choice questions gathered specifics like before and after preparation times, while our open-ended questions gave participants space to declare reasons and explanations. For example, we asked "Given a choice, which computer do you usually use to prepare medication? Why?" and "Do you encounter problems when using a COW? What kind of problems and how often do you encounter them?"

We used open coding to analyze the observation and interview data to identify and group common themes. Our findings were verified by several participant nurses. We tabulated the survey results to look for similarities and differences.

\section{Participants}

In the early stage, study participants were 2 patient care managers, 35 registered nurses, and 1 nursing student. In the later stage, study participants in our observations were 1 patient care manager, 24 registered nurses, and 2 nursing students. 29 nursing staff participated in the survey.

All our participants were working on the ward at the time of the study. All our questionnaire respondents had personal experiences in retrieving and entering information in the EHR. Since the participants use the technology to fulfill their job, they were highly motivated to provide feedback and their personal experiences with the technology.

\section{FINDINGS}

With the new technology, the EHR became the primary information source and repository for nursing care. Nurses no longer assemble inside the shift change room during shift changes. Instead, they prepare their shift at a computer, either at a wired desktop computer or a wireless COW. Given a choice, most of them would use a desktop computer inside the computer terminal room. However, due to organizational design, nurses can only use the computer terminal during night/day $(\sim 7 \mathrm{am})$ and evening/night $(\sim 11 \mathrm{pm})$ shift changes because physicians generally occupy

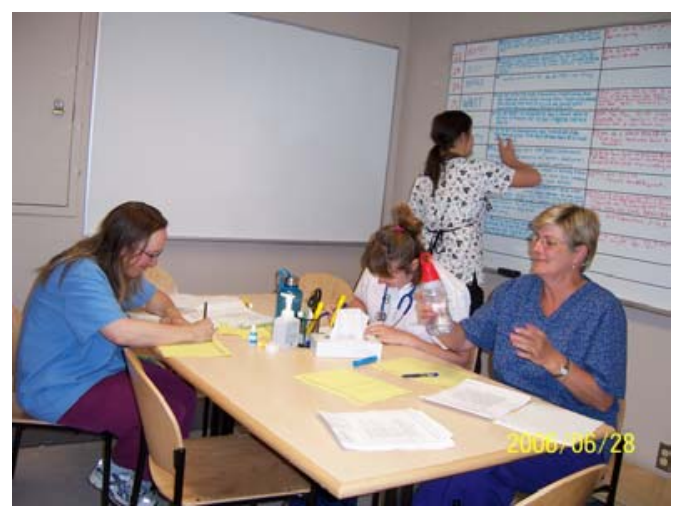

Figure 5. Nurses preparing for their shift while socially interacting inside the shift change room before deployment of the COW this space during day/evening shift change ( $\sim 3 \mathrm{pm})$. Thus this space is commonly referred as the "physicians' area". Nurses might sometimes work at a desktop computer in a ward wing; they would have to either stand or sit on a high backless stool to use these machines (Fig. 2).

The star configuration of the ward layout spatially separates nurses during their shifts. The spatial distribution often makes them unaware of what was going on in other wings. Therefore, most nurses rely on the opportunity to get together for social interaction during shift changes (Fig. 5).

\section{Early Stage of Technology Deployment}

In the early stage of the technology deployment, nurses were highly encouraged to use the COW for information access and entry although stationary desktop computers were still available along ward wings and inside the computer terminal. They were also motivated to experiment with the new technology as they believed that the technology could help improve their work performance. Yet, we observed several undesirable impacts as a result of the technology deployment (briefly reported in [22]).

Virtual mobility. The COWs were designed to be mobile with the ergonomically designed cart so that they could be easily moved for use at different points of care. However, only one of the nurses we observed would always bring a COW into patient rooms and enter medical information directly to the EHR without first transposing to her note sheet. Yet, she still kept a personal worksheet of important medical information with her as a reminder and quick reference. All other nurses said they rarely brought a COW to points of care because of its bulkiness and clumsiness. Also, the computer screen and keyboard did not provide the same affordances as paper and pen. With paper, they could easily and quickly jot down notes whereas with the COW, they first had to $\log$ on and to navigate to the right screen of the information system, then type information in the rigidly formatted cells. They found this activity too timeconsuming. Therefore they would first write information down on their paper note-sheet and only enter it into the EHR when they had time or at the end of their shift.

Moreover, many rechargeable batteries wore down quickly so that nurses were asked to always plug in the COWs whenever they were not being used. Some nurses found this tedious and time-consuming. An experienced nurse commented, "Recharging the COW is not a priority of my job!" Therefore the nurses were generally hesitant about using the COW due to the additional overhead required.

Less time for patient care. The patient care summary which was used before the adoption of the COW was an abbreviated EHR containing updated patient's medical information at the beginning of each shift. Nurses had usually been able to get a good grasp of their patient's condition and to devise an adequate shift-specific nursing care plan for each patient after reading the patient care summaries and the displayed shift report on whiteboards, 
while more detailed information could be found in the full digital record [21]. However, the new EHR always displayed the patients' complete medical information. Thus, the time required to read through the extensive record was considerably longer. This was exacerbated by the large number of finely specialized categories of information residing in specific windows in the new information system that they had to navigate to the right screen before finding the necessary information. All nurses we observed required more time with the new system; several nurses commented that they needed twice as much time as before.

Similarly, outgoing nurses needed more time to chart new information into the EHR as they were not yet familiar with the new system and the deep hierarchical information structure also complicated the process. We observed a nurse trying to chart the nutritional intake of a patient. She could not remember where the information should be placed. She asked another nurse who was nearby. They tried the keyword search for "nutrition", "diet", and "food intake", but none was right. The nurse then decided to just put that information as part of the online nurse-to-nurse communication so that at least the incoming nurse would know about the information.

With the increased time needed in both preparation and charting for a shift, there was less time left for delivering patient care unless the nurses stayed behind after their shift. Indeed many nurses stayed longer during our observations.

Possible compromise of patient care. With the known volatile battery life and a concern for confidential patient information being disclosed if nurses forgot to $\log$ themselves off the system, the COWs were set to run on power-save mode such that the system would turn off automatically after a few minutes' idling. This has proved to be a considerable challenge for nurses when they were preparing medications. The monitor displayed medication information while nurses prepared them at a medication cart (a.k.a. med-cart). Nurses always placed a COW beside the med-cart. Their hands were usually occupied with medicine and apparatus such as a syringe and while the computer remained idle in terms of input, the nurses were following the on-screen information. Therefore, the fear of getting logged off by the system often compelled the nurses to subconsciously rush to complete the preparation. This is because each time they were logged off, not only would they need to $\log$ on the system again, they also had to manually navigate to the right screen before they could continue the task. Such unintentional hasty behavior unfortunately can be prone to adverse events, in particular, medication error which has been found to be one of the most common preventable non-operative (i.e. non-surgical) adverse events [21].

Users' well-being jeopardized. The considerable amount of time that nurses must spend at a computer when working with the EHR brought out other issues that were not as pronounced before the implementation of the new technology. One such issue is the seating comfort, or more appropriately seating discomfort, caused by the high wooden backless stools (Fig. 4, left). Since having to use these stools, many nurses reported that they frequently suffered from back and shoulder pain that forced them to be absent from work. This unfortunately aggravates the nurse shortage problem already experienced in the local health region. Also, coupled with the heavy mouse manipulation necessary to navigate the deep hierarchical information system, conventional computer hardware posed added difficulty to right-handed people. Right-handed nurses frequently had to switch their dominant hand between a mouse and a pen while preparing for their shift. This increased both preparation time and muscle fatigue.

Moreover, with the new technology, nurses no longer gathered inside the shift change room (Fig. 5). Instead, they spent most of their time during shift changes at a computer reading medical reports of their patients. They either used a COW which was usually parked along the ward wings or a computer inside the computer terminal depending on its availability. When reading reports in a hallway, nurses were usually distributed making it almost impossible to socialize with their colleagues. While the COWs offered mobility, nurses actually felt more distant from their colleagues because they were all scattered over the ward wings and were usually too busy with the information system. Thus, they much more rarely found opportunities for social interaction. Even when they could gather inside the computer terminal, the set-up there did not support interaction, especially because they had to focus at the computer screen in front of them (Fig. 3). Therefore, many nurses complained that they missed the rich social interaction that used to take place with their colleagues inside the shift change room (Fig. 5). Since using the COWs, a nurse commented, "very often I felt so lonely, it's like um' I'm the only one here... I don't like it..."

Continued use of paper notes. Although nurses were expected to directly interact with the COW for information access and entry at points of care without using intermediary artifacts, all nurses we observed still prepared a paper-based personal note-sheet. They carried and used these worksheets during their shift as an immediate information source and an intermediary notepad, in the same way as before the COW was deployed. Most of them only made adjustments to the layout of the note-sheet and extracted more information from the EHR to the personal notes to make up for the unavailability of frontline information provided by the phased-out patient care summaries. In fact, they all commented that the COW could not replace their personal note-sheet which allowed them to easily find information they needed and that it was a crucial part of their work practice [22]. 
Despite all the difficulties confronted by the nurses in the early stage of the technology deployment, most of them were hopeful that the difficulties would subside over time.

\section{Later Stage of Technology Deployment}

Eleven months after the initial deployment of the technological set-up, we followed up with another observation to uncover longer-term phenomena. We observed improvements in some of the problems identified in the early stage, some problems that persisted or worsened through the adoption period, and some new problems that had emerged over the longer period of time.

\section{Observed Improvements}

Workarounds to improve quality of patient care. Nurses were found to adapt better with workarounds to the powersave log-off problem identified in the early stage of adoption. Some of the nurses would arbitrarily move the mouse when being alerted audibly (the last 10 seconds) in order to reinstate the timer for idleness. Thus the frequency that nurses were being signed off by the power save feature had been reduced. Nevertheless some nurses still found this distracting from their task-at-hand.

Regained time for patient care. Nurses have become more familiar with the new information system over time and use. Most problems that they encountered when retrieving or entering information in the early stage of adoption have been improved. They generally had better knowledge of where specific information resided and were also more familiar with the terminology in the system. Nurses told us that they were now faster at reading and charting with this system and this speed-up was clearly noticeable in observations. Thus nurses, in comparison to the early stage observations, were now able to spend more time on delivering patient care.

Nurses actively combat deteriorating social interaction. Nurses were found to engage in social communication more frequently inside the computer terminal during night/day and evening/night shift changes. With the existing physical set-up, nurses had to turn their body around in order to engage in conversations with nurses sitting on the opposite side of the terminal (Fig. 3). Yet, as most nurses had realized the fading social interaction since the technology adoption, they therefore put in conscious effort to improve the communication among themselves.

\section{Persistent or Worsened Problems}

Increased Immobility. The COWs were intended for mobile use, however, more problems were identified that contributed to its failure to fulfill this anticipated use. These problems included unreliable network connectivity, suboptimal battery life, and unsatisfactory ergonomics.

First, the connectivity problem was attributed to either the wireless network being unstable or the presence of dead zones in scattered areas on the ward. Unstable wireless connection was often experienced while nurses were

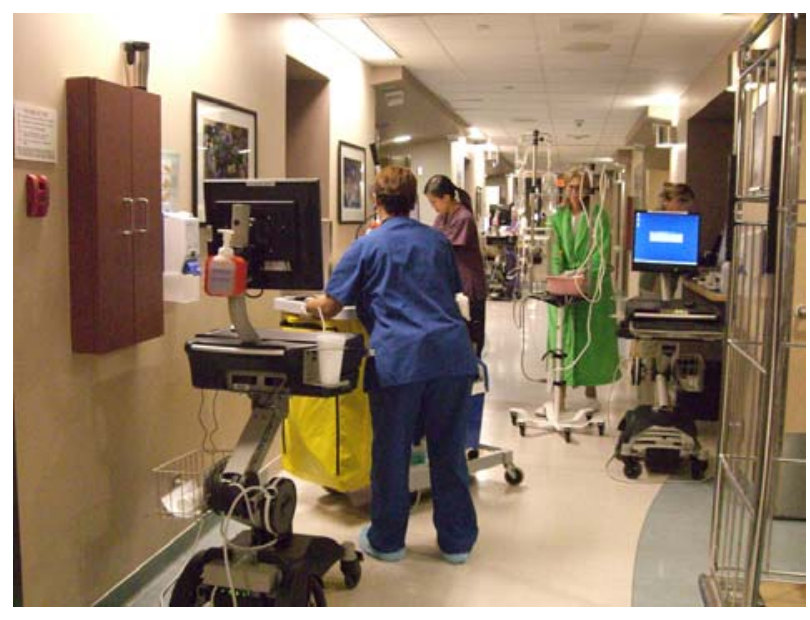

Figure 6: A crowded hallway parked with the COWs making it difficult for patients to go through or janitors to carry out their work

working on a COW. Sudden log-offs required nurses to re$\log$ on and to tediously navigate to the last visited page in order to continue their task. Other times, when nurses moved a COW to use, e.g., in a patient room, connectivity might not be available. Some nurses would try to slightly shift the device back and forward or at another orientation in an attempt to get connectivity when they were not too time-pressured. Most nurses would just give in and resort to more reliable paper artifacts, specifically their personal notes for information and as a notepad.

Second, more batteries were reported as wearing down too quickly and the COWs were constantly required to be plugged in for recharging. A nurse responded in the questionnaire, "I like to take the COW into patient room but they ALWAYS need to be plugged in if using them for any length of time longer than 5 minutes..." Thus with such short battery life, either the task-at-hand got interrupted when the battery ran out or nurses would subconsciously, similar to the medication preparation reported in the early stage of deployment, rush through the task. The former would frustrate the nurses and impede the work flow whereas the latter might result in serious adverse consequence such as medical errors.

Third, the physical attributes of the COWs remained unchanged. Since the carts were bulky and clumsy, the COWs were counterintuitive as a mobile device. Figure 6 shows a crowded hallway where pushing a COW is no easy task especially when there were other people, structures or equipment present such as slow-moving patients or a janitor with a cleaning cart.

While five (out of 29) nurses said they almost never brought the COW into patient rooms, only one said she almost always brought it to patient rooms, except for isolated rooms. Most other nurses (23/29) either sometimes or occasionally brought the COW into patient rooms for varying reasons such as in the initial rounds of assessment, 
critical events (e.g., treating a dying patient), performing certain procedures (e.g., blood transfusion) and explaining complicate medications. Nurses also explained that they rarely brought the $\mathrm{COW}$ into patient rooms at night as its bright screen could disturb the patients' sleep.

Declining well-being. Nurses continued to complain about the back and shoulder pain problem caused by the inappropriate seats. Regardless, they still preferred sitting to standing for reading or charting the EHR. Many nurses expressed bitter comments towards the seating facilities, e.g., "I refuse to stand or sit on stool, it hurts lower back, no back support with stools, also hard on feet..."

\section{Newly Emerged Problems}

Weakening interpersonal communication. Many nurses found the COWs impede communication with their patients because of its size and rigidity. The computer appeared less "human" and more intrusive during interpersonal communications. Since information can only be entered in a constrained way at a computer, it is not as flexible as the paper medium which allows easy interweaving with the ongoing assessment and explanation of procedures and medication. COWs seemed to interfere with comfort and sentimental work (as defined in [20]).

Mechanical flaws. Nurses found the mobile carts difficult to steer around because of sticky or misaligned wheels, similar to what many of us have experienced with "difficult" shopping carts in supermarkets. Some nurses also complained about the difficulty of adjusting the height of the monitor and keyboard as well as the mechanical stickiness of the keys. As a nurse's job is always timepressured, they resented having to deal with these technology-related obstacles.

Role-models not conforming to organizational goal. The technology deployment was planned for pioneering the ward towards paperless. However, we observed several mentoring sessions in which the mentors, who were usually experienced nurses, were teaching their "students" to prepare paper-based personal notes by extracting information from the EHR. This continuing use of paperbased artifacts as the communication tool for reporting and at meetings was observed with all our participants.

Frustrated adopters. With the problems described above, some of the nurses have become frustrated with the technology. Several nurses even explicitly said that they would avoid using the COWs whenever possible. We encountered an incident where a nurse was looking for a computer to read reports at the beginning of her shift. The nurse refused to use any of the COWs idling along the hallway. Rather, she walked around the ward to try to find a desktop computer. It was day/evening shift change so desktop computers were not available in the computer terminal. When a nursing aid suggested to her to use a COW, she simply snapped, "No, they never work!" She finally settled down at a desktop computer at the nursing station after checking all the ward wings for unoccupied desktops.

Our study found that nurses had a strong preference for desktop computers over the COWs. $96 \%$ of our questionnaire respondents indicated that desktop computers were their most preferred device for reading and charting reports during shift changes. The primary reasons for the choices were based on the wired network stability and performance, as well as the seating comfort adherent with most of the desktop computers. Most nurses criticized the wireless connection as unstable and as causing different kinds of problems. These problems started from the sign-on process which often failed, or required several trials or sometimes required rebooting of the system. Then intermittent freezing and complete disconnection from the system was frequent in the course of working with the information system. Thus, most nurses were reluctant to work with the COW particularly when they needed to use a computer for a longer period of time such as when they read reports at the beginning of a shift or charted at the end of their shift. In addition, the wireless network was generally slow and sometimes unresponsive. Nurses complained that page loading was sometimes so slow that they had to halt the task at hand and only returned to retry when they had time later. In these respects, desktops offered more consistent performance and they were generally faster. Thus, nurses would always prefer desktops over the slow mobile COW, especially in view of the time pressure that nurses constantly face.

In the course of our data analysis, we noted a considerable amount of negative feedback on the COW adoption, mostly due to their unsatisfactory technical performance and their lack of consideration for human factors. We thought that our questionnaire respondents would not recommend deploying the same technology in other wards. However, surprisingly 15 of 28 respondents unconditionally recommended the use of the COWs in other hospital wards and six respondents conditionally recommended the technology. The conditions were all expected improvements to solve the problems described in this section. Only four were firm on not recommending the technology to other wards. We were intrigued by the questionnaire responses. Thus, we reviewed the raw data again and randomly interviewed some nurses. We found that there were not enough computers prior to the deployment of the COWs. Nurses often had to wait for their turn to use a computer. Therefore, despite the flaws with the technology, they still opted for their adoption in other wards as they believed that the devices would add valuable resources to other wards. The deeper connotation is that if it is decided that the COWs are to be removed, they will then lose these valuable computing resources now available to them. Therefore to the nurses, the COWs provided extra information devices regardless of the adjustments they may have to make in order to compensate for their flaws. 
For the same reason, COWs were most preferred for preparing medication. Prior to adoption of the new technology, a medication list would be printed out for each nurse on an hourly basis to prepare medication at the medcart and multiple nurses could work at a med-cart simultaneously. But medication information is now only available on screen with the new information system. As each ward wing was served by only one med-cart, it would be inconsiderate to bring the med-cart to a desktop computer to prepare medication. In such case, only one nurse could use the med-cart at any one time. With the COWs, multiple nurses could share a med-cart by placing their COWs beside it. Hence, this again showed that the COWs added valuable resources to the nurses.

\section{DISCUSSION}

Our study revealed an array of difficulties that nurses encountered either directly or indirectly from the deployment of the COW in our study ward. As a result, many of our participants either completely abandoned or tried to avoid using a COW. These study results can be used to either suggest changes for the COWs or point to new design directions.

\section{Issues to Resolve with COW Use}

As the COWs do offer valuable extra computing resources to nurses' shift work, it is important that the shortcomings identified be addressed. The technical and engineering problems associated with the mobile device require appropriate technical support team servicing. The batteries should be regularly tested and recharged to ensure that they function for a reasonable time period, e.g., 24 hours, without interruption and it seems reasonable that this not be considered to be part of a nurse's job. Faulty batteries should be replaced. Technical support servicing should also ensure reliable network connectivity throughout the deployment area. Thus, decreasing observed problems with deployment of a $\mathrm{COW}$ requires adequate funding for required technical support servicing.

The physical form of the device should be re-thought, new versions could be improved and the ergonomic factors that influencing nurses' experience with the device could be addressed. Currently, this includes the maintenance of various engineering parts such as the height adjustment, the wheel alignment and smoothness, as well as the sensitivity of the input keys.

The social concern experienced by the nurses also needs to be addressed as social interaction has been found to be crucial for collaborative work. A solution to resume social interaction during shift changes is to equip the shift change room with wired notebook computers so that nurses can continue sitting around a large table to prepare for their shift while casually communicating with each other. This set-up should also alleviate nurses' back and shoulder pain problems, which in turn help ease the short-staffed issue.
Nevertheless, we are more interested to find out why most nurses stated that the mobile device cannot replace their paper personal notes in delivering nursing care. Even if we assume that the identified technical, engineering, and social problems engendered by the COW deployment can be addressed, it appears that there still will remain problems associated with the use of and advantages offered by paper based artifacts. From our studies [21,22,23], we observed the construction of these paper artifacts. We interviewed nurses to find out when, where, and how these artifacts were used during their shift. By examining the paper artifacts that we collected at the beginning and at the end of their shifts allowed us to trace how these artifacts were used during their shifts. We also observed their use at reporting and handover.

\section{Paper Personal Notes vs. COW}

In this section, we compare observed functionality of paper based artifacts for mobile information access with the COW as a mobile information source. The paper artifacts are personally created so they display a high degree of personalization (see [21] for details). We also identified several important roles that these paper artifacts play in the nurses' actual work practices (see [23]). The paper artifact was used to:

- hold the work plan for delivering patient care,

- provide a bedside information source,

- be an opportune notepad, for recording information, and

- be an information source for reporting and handover

\begin{tabular}{|l|l|}
\hline \multicolumn{1}{|c|}{ Paper personal notes } & \multicolumn{1}{c|}{ COW } \\
\hline $\begin{array}{l}\text { Foldable and portable in pockets, } \\
\text { so low cost }\end{array}$ & $\begin{array}{l}\text { Wheeled cart for mobility, } \\
\text { difficult in crowded space }\end{array}$ \\
\hline $\begin{array}{l}\text { Customizable as work plan, } \\
\text { overview visualization }\end{array}$ & $\begin{array}{l}\text { Information scattered in } \\
\text { different screens }\end{array}$ \\
\hline $\begin{array}{l}\text { Manual construction help build } \\
\text { mental map }\end{array}$ & $\begin{array}{l}\text { Memory overload of } \\
\text { information }\end{array}$ \\
\hline $\begin{array}{l}\text { Convenient, low-cost bedside } \\
\text { information source }\end{array}$ & $\begin{array}{l}\text { High cost of information } \\
\text { access }\end{array}$ \\
\hline $\begin{array}{l}\text { Flexible, low-cost immediate } \\
\text { notepad }\end{array}$ & $\begin{array}{l}\text { High cost of information } \\
\text { entry }\end{array}$ \\
\hline $\begin{array}{l}\text { Centralized, overview information } \\
\text { basis for reporting and handover }\end{array}$ & $\begin{array}{l}\text { Information scattered in } \\
\text { EHR or memory overload }\end{array}$ \\
\hline
\end{tabular}

Table 1. A comparison of paper personal notes and the COW

Table 1 shows how these vital roles are realized with paper based artifacts (left-hand column) and with the COW (right hand column). Note the sharp difference between the flexibility and mobility provided by paper in contrast to how the COW has failed to live up to its intended use as a mobile and ubiquitous information artifact in nursing care. Next, we discuss these roles in more detail and subsequently offer design guidelines. 


\section{Work Plan}

Information recorded in the personal notes includes action items such as reminders and to-dos, alerts, prompts, scheduling, and verification information. The manner in which these information types is presented on the personal notes, is often customized through visual augmentations such as highlighting, annotations, special signs and icons, color coding and spatial layout. These visual augmentations inform a rich set of meanings in addition to plain medical facts. When nurses prepare their personal notes, they cognitively make plans for the temporal performance of the tasks that need to be done during their shift. Also, the manual writing of information on their personal notes helps them build a strong mental map of their patient's condition and their shift work. Thus the notes inform them of the tasks to be performed, the order in which the tasks should be carried out and an overview of their shift work.

In contrast, the COW does not offer the same affordances. As information displayed on a COW cannot be customized, nor can specific information be extracted and placed separately for personal use, nurses relying on the COW must memorize the information they read. Alternatively, they will have to frequently access information to make sure the tasks are carried out as required and in the correct temporal sequence. Therefore, the COW does not provide work plan support for the nursing shift work.

\section{Bedside Information Source}

Paper personal notes are portable and malleable. They can be folded and put in a pocket or conveniently placed on a clipboard for easy writing, thus can provide customized information at points of care. The customized notes allow nurses to quickly look for specific information. Therefore the information access process is light-weight and can also be easily interwoven with other tasks or a conversation.

While the COW also allows information access at bedside, the cost of the process is high. Nurses have to log on to the system before they can navigate to the appropriate screen for required information through mouse manipulation. As nurses may have to look up information frequently during their shift work, this high cost of information access is likely substantial considering the constant time pressure that nurses face at work. Besides, with the physical barrier of the computer screen, keeping constant eye-contact with patients or maintaining a conversation while searching for information in a COW is no easy task.

\section{Opportune Notepad}

During nursing work, new data emerges frequently and ubiquitously especially for unstable patients. Nurses often first record the emerging information in their personal note as a means to reduce their mental workload and eventually report it in official documents of varying media. Personal notes are generally pre-structured at its point in time. Nurses thus can quickly scribble new but anticipated data in specific information holders on their personal notes, while they can also casually add the new and unanticipated information in available open space not specified in the usual spatial layout of their personal note. Thus the personal notes allow nurses to flexibly and speedily scribble notes and annotations as a temporary repository.

On the other hand, nurses cannot use the COW in the same way to quickly or easily scribble down newly emerged information. Nurses either have to rely on their mental capacity or first access the information system and then type in the new information using a $\mathrm{COW}$ at points of care. The former easily overloads the nurses' memory and it also runs the risk of them forgetting information that is important in the patient care. The latter is problematic due to the high cost of information entry. It undoubtedly further strains the time-pressured nursing work.

\section{Information Basis for Reporting and Handover}

New information added to the personal notes during a shift often forms part of a patient's illness trajectory. Therefore, such information must be properly documented for use by other clinicians and for later review when needed. Nurses are required to report their work at the end of a shift, to the succeeding nurse and to the charge nurse. Their personal notes, containing the newly emerged information which also represents the patient's shift-specific illness trajectory, thus serve as important information basis for reporting.

Using a COW to display information during reporting can be a challenge as information is scattered in the hierarchical information system. Nurses either have to navigate, at high costs, to different screens for finding the right information to report or rely on their memory which again may result in reporting or handover of incorrect information due to memory lapses. Focusing on the computer screen also makes it difficult for keeping eye contacts with colleagues.

\section{Design Suggestions}

We discussed the COW's failure to replace the paper personal notes in nurses' shift work in our study ward. We suggest that paper will continue to play an integral part in nurses' work practices due to its unique affordances. Thus a potential future technology design could focus on how the paper and the digital world may be bridged so that information can be converted efficiently between the divide. Therefore we propose that the system should:

- allow easy transposing of information from multimedia sources to a paper-like physical artifact,

- allow portable, flexible and low-cost use of a paper-like artifact to support and enhance work performance, and

- allow effortlessly moving information from the paperlike artifact back to the multimedia sources to provide timely, low-cost and continuous information flow.

\section{CONCLUSION AND FUTURE WORK}

Our study to investigate the impacts of a mobile technology deployed in a local hospital ward identified short-term and long-term problems encountered by the nurses. We proposed solutions to address the identified technical, engineering and social problems. However, we directed our 
attention to the mismatch between the deployed mobile technology and the nurses' current work practices in our study ward. We compared the COW to the paper personal notes in terms of the latter's roles in current nursing practices. We also described our design suggestions for future technology to support the shift work. Our next step is to prototype our design ideas and to evaluate its effectiveness in enhancing nurses' shift work.

\section{ACKNOWLEDGMENTS}

We are indebted to the nursing staff on the W21C at the Foothills Hospital for their incredible support. We also thank AIF, iCore and NSERC for their generous funding.

\section{REFERENCES}

1. Bardram, J. \& Bossen, C. Mobility Work: The Spatial Dimension of Collaboration at a Hospital. JCSCW 2005, 14(2): 131-160.

2. Bardram, J. Temporal Coordination: on time and coordination of collaborative activities at a surgical department. JCSCW 2000, 9(2): 157-187.

3. Bellotti, V. \& Bly, S. Walking Away from the Desktop Computers: distributed collaboration and mobility in a product design team. Proc. of CSCW 1996, pp. 209-218.

4. Cabitza, F., Sarini, M., Simone, C. \& Telaro, M. When Once Is Not Enough: the role of redundancy in a hospital ward setting. Proc. of GROUP 2005, pp. 158167.

5. COWs help sick children: Case studies and profiles. http:/www.smh.com.au/news/case-studies-profiles/cows-help-sickchildren/2007/03/05/1172943353087.html. 3-30-2008.

6. Gurses, A. \& Xiao, Y. A Systematic Review of the Literature on Multidisciplinary Rounds to Design Information Technology. Journal of the American Medical Informatics Association, 13(2) 2006: 267-276.

7. Harper, R., O’Hara, K., Sellen, A. \& Duthie, D. Toward the Paperless Hospital? British Journal of Anaesthesia. 78, 1997: 762-767.

8. Heath, C. \& Luff, P. Documents and Professional Practice: 'bad' organizational reasons for 'good' clinical records. Proc. of CSCW 1996, pp. 354-363.

9. Kane, B. \& Luz, S. Multidisciplinary Medical Team Meetings: an analysis of collaborative working with special attention to timing and teleconferencing. JCSCW 2006, 15:501-535.

10. Kovalainen, M., Robinson, M. \& Auramaki, E. Diaries at Work. Proc. of CSCW 1998, pp.49-58.

11. Kraut, R., Egido, C. \& Galegher, J. Patterns of Contact and Communication in Scientific Research Collaboration, Proc. of CSCW 1988, pp.1-12.

12.Lu, Y., Xiao, Y., Sears, A., \& Jacko, J. A Review and a Framework of Handheld Computer Adoption in
Healthcare. Int'l J. of Med Informatics 74(5) 2005: 409422.

13. Luff, P. \& Heath, C. Mobility in Collaboration. Proc. of CSCW 1998, pp. 305-314.

14.Luff, P., Heath, C., \& Greatbatch, D. Tasks-inInteraction: paper and screen based documentation in collaborative activity. Proc. of CSCW 1992, pp. 163170.

15. Moran, E., Tentori, M, Gonzalez, V., Favela, J. \& Martinez-Garcia, A. Mobility in Hospital Work: towards a pervasive computing hospital environment. International Journal of Electronic Healthcare, 3(1) 2007: 72-89.

16. Nomura, S., Hutchins, E. \& Holder, B. The Uses of Paper in Commercial Airline Flight Operations. Proc. Of CSCW 2006, pp. 249-258.

17. Reddy, M. Dourish, P. \& Pratt, W. Temporality in Medical Work: time also matters. JCSCW 15(1), 2006: 29-53.

18. Sellen, A. \& Harper, R. The Myth of the Paperless Office. The MIT Press, Cambridge, Massachusetts, London, England, 2002.

19.Skov, M. \& Høegh, R. Supporting Information Access in a Hospital Ward by a Context-aware Mobile Electronic Patient Record. Pers and Ubiq Computing 2006, 10(4): 205-214.

20.Strauss, A. Fagerhaugh, S., Suczek, B. and Wiener, C. Social Organization of Medical work. The University of Chicago Press, 1985.

21. Tang, C. \& Carpendale, S. An Observational Study on Information Flow during Nurses' Shift Work. Proc. of CHI 2007, pp. 219-228.

22. Tang, C. \& Carpendale, S. Impacts of Technology Deployment on Information Assembly and Disassembly during Shift Change. Workshop on Handover: collaboration for continuity of work, ECSCW 2007.

23. Tang, C. \& Carpendale, S. Support for Informal Information Use and its Formalization in Medical Work. Proc. of IEEE CBMS 2008, pp. 476-481.

24. Weingart, S.N., Wilson, R., Gibberd, \& Harrison, B. Epidiomiology of Medical Error. British Journal of Medicine 2000, 320: 774-777.

25. Whittaker, S., Frohlich, D. \& Daly-Jones, O. Informal Workplace Communication: what is it like and how might we support it? Proc. of CHI 1994, pp. 131-137.

26. Wilson, S., Galliers, J., \& Fone, J. Not All Sharing is Equal: the impact of a large display on small group collaborative work. Proc. of CSCW 2006, pp. 25-28.

27.Xiao, Y., Lasome, C., Moss, J. \& Mackenzie, C. Cognitive Properties of a Whiteboard: a case study in a trauma centre. Proc. of ECSCW 2001, pp. 259-278. 